\title{
Effect of iron saturation level of lactoferrin on osteogenic activity in vitro and in vivo
}

\author{
X. Y. Wang, ${ }^{* 1}$ H. Y. Guo, ${ }^{* 1}$ W. Zhang,† P. C. Wen, $\ddagger$ H. Zhang,§ Z. R. Guo, ${ }^{*}$ and F. Z. Ren ${ }^{* 2}$ \\ *Key Laboratory of Functional Dairy, and \\ †Beijing Higher Institution Engineering Research Center of Animal Product, College of Food Science and Nutritional Engineering, \\ China Agricultural University, Beijing 100083, China \\ ¥College of Food Science and Engineering, Gansu Agricultural University, Lanzhou 730070, China \\ $\S B e i j i n g$ Key Laboratory of Nutrition, Health and Food Safety, Beijing 100083, China
}

\section{ABSTRACT}

We studied the effect of iron saturation level on the osteogenic activity of lactoferrin (LF) in vitro and in vivo. Different iron saturation levels of LF (1.0, 9.0, 38, 58 , and $96 \%$ ) were prepared as the following samples: apo-LF, LF-9, LF-38, LF-58, and holo-LF. Using the 3-(4,5-dimethylthiazol-2-yl)-2,5-diphenyltetrazolium bromide (MTT) assay, we observed that the stimulating osteoblast proliferation activity of LF in vitro decreased with increasing iron saturation level at 100 and $1,000 \mu \mathrm{g} / \mathrm{mL}$. In vivo, 4 -wk-old ICR Swiss male mice were randomly divided into 4 groups: blank control (physiological saline), negative control (BSA), apo-LF, and holo-LF. Four groups of mice were injected subcutaneously with physiological saline, BSA, apo-LF, or holo-LF over the calvarial surface twice a day for 5 consecutive days at a dose of $4 \mathrm{mg} / \mathrm{kg}$ per day. Bone histomorphometry showed that new bone formation (assessed using tetracycline- $\mathrm{HCl}$ labels) tended to be stronger with apo-LF than with holo-LF. Using fluorescence spectroscopy and circular dichroism measurements, we found that exposure of tryptophan increased, $\alpha$-helix content increased, but $\beta$-structure content decreased with increasing iron saturation level. These findings indicated that the osteogenic activity of LF decreases with increasing iron saturation level in vitro and in vivo, which may be related to conformational changes in LF.

Key words: lactoferrin, iron saturation level, osteoblast, bone

\section{INTRODUCTION}

Lactoferrin ( $\mathbf{L F})$, an $80-\mathrm{kDa}$ iron-binding glycoprotein of the transferrin family, is produced by epithelial

\footnotetext{
Received May 4, 2012.

Accepted October 6, 2012.

${ }^{1}$ The first 2 authors contributed equally to this paper.

${ }^{2}$ Corresponding author: renfazheng@263.net
}

cells and neutrophilic leukocyte precursors (Metz-Boutigue et al., 1984). Lactoferrin is widely distributed in body fluids (Lönnerdal and Iyer, 1995) and is present at very high concentrations in colostrum and milk (Nagasawa et al., 1972; Sánchez et al., 1988). Lactoferrin is a pleiotropic factor that exhibits a vast range of biological functions. Recently, it has been demonstrated to be an anabolic factor for bone in vitro and in vivo. Studies in vitro proved that LF potently stimulated the proliferation and differentiation of osteoblasts (Cornish et al., 2004). Furthermore, local administration of LF over the calvariae of adult male mice (Cornish et al., 2004) indicated that LF increased bone formation in vivo. Oral feeding trials in ovariectomized rats (Blais et al., 2009; Guo et al., 2009) have indicated the potential of LF in improving bone density. Compared with the majority of current medications for osteoporosis, LF not only inhibits bone resorption but also has the ability to increase the activity of osteoblasts and bone formation. Thus, LF is a promising therapeutic target in bone repair and osteoporosis.

Lactoferrin is a member of the transferrin family and an important regulator of the levels of free iron in the body fluids of mammals (Sawyer and Holt, 1993). In general, $\mathrm{LF}$ has the ability to bind $2 \mathrm{Fe}^{3+}$ very tightly but reversibly (dissociation constant $K_{\mathrm{d}}$ $\sim 10^{-22} M$; Baker and Baker, 2004). Therefore, LF has different $\mathrm{Fe}^{3+}$ binding statuses: the iron-free (apo) form, the native form, and the iron-saturated (holo) form. From biophysical studies, it is known that apoLF is less stable and less compact than holo-LF, and a large conformational change accompanies iron binding and release (Grossmann et al., 1992). Crystallographic studies on human apo-LF have shown that the release of iron causes one domain to swing away from the other to open up the binding cleft (Anderson et al., 1990). These studies all indicate that iron binding affects the conformation of LF. Moreover, several studies have suggested that LF with different iron saturation levels exert different physiological functions. Some studies have 
shown that the resistance of LF to denaturation and proteolysis increases with an increase in iron saturation level (Anderson et al., 1990; Chan and Li-Chan, 2007; Lönnerdal et al., 2011). Bullen et al. (1972) showed that the antibacterial activity of LF decreased with an increase in iron saturation level. It has been reported that apo-recombinant human LF possessed a stronger growth inhibiting effect compared with holo-recombinant human LF on HT-29 cells (Huang et al., 2008). And Jiang and Lonnerdal (2012) reported that the effect of apo-LF on stimulating proliferation of crypt cells was significantly stronger than that of holo-LF in vitro. However, the effect of iron saturation level on the osteogenic activity of LF remains unclear.

Therefore, the objective of our study was to investigate the effect of iron saturation level on osteogenic activity, including osteoblast proliferation activity in vitro and new bone formation of LF in mice. Circular dichroism and intrinsic tryptophan fluorescence were used to evaluate conformational changes of LF induced by iron saturation level.

\section{MATERIALS AND METHODS}

Lyophilized bovine LF with 95\% purity (SDS-PAGE) was supplied by Australian Yosica Holding (Melbourne, Australia). The SDS-PAGE electrophoretogram showed that angiogenin $(\sim 16 \mathrm{kDa})$ was undetectable. The contents of endotoxin and transforming growth factor- $\beta$ in the sample were $0.22 \times 10^{-3}$ endotoxin units (EU)/ $\mathrm{mg}$ of $\mathrm{LF}$ and $1.3 \mathrm{ng} / \mathrm{mg} \mathrm{LF}$, respectively, as determined by ELISA kits (R\&B Company, San Jose, CA). Lactoferrin was dialyzed against 30 volumes of Milli$\mathrm{Q}$ water (Millipore, Billerica, MA). The red solution was freeze-dried and stored at $-20^{\circ} \mathrm{C}$. Ferric chloride, nitrilotriacetic acid (NTA), and citric acid (Sinopharm Chemical Reagent, Shanghai, China) were of analytical grade. Dialysis membranes (Viskase, Darien, IL) had a molecular weight cutoff of $14 \mathrm{kDa}$.

\section{Preparation of Bovine LF with Different Iron Saturation Levels}

Iron-depleted (apo) LF was prepared by titrating a $1 \%$ solution of LF in Milli-Q water (Millipore) to $\mathrm{pH}$ 2.2 with $\mathrm{HCl}$, and then dialyzing it 4 times against 30 volumes of $0.1 \mathrm{~mol}$ of citric acid/L $(\mathrm{pH} 2.3)$ at $4^{\circ} \mathrm{C}$. Citrate was removed using dialysis against 30 volumes of Milli-Q water (fourth renewed) at $4^{\circ} \mathrm{C}$, and conductivity was metered to ensure the stability of conductivity. The resulting colorless solution was freeze-dried and stored at $-20^{\circ} \mathrm{C}$. Iron depletion was confirmed spectrophotometrically (Bates and Schlabach, 1973).
Other iron saturation forms of LF were prepared by dissolving LF (1\% protein, wt/vol) in $10 \mathrm{mM}$ Tris- $\mathrm{HCl}$ buffer containing $75 \mathrm{mM} \mathrm{NaCl}$, at $\mathrm{pH}$ 7.2. This solution was then mixed with different doses of freshly prepared FeNTA solution $\left(9.9 \mathrm{mM} \mathrm{FeCl}{ }_{3}\right.$ and $8.5 \mathrm{~m} M$ NTA, pH 7.0) and incubated for $1 \mathrm{~h}$. Excess reagent was removed using dialysis against 30 volumes of Milli-Q water (fourth renewed) at $4^{\circ} \mathrm{C}$. These red fractions containing $\mathrm{LF}$ were then freeze-dried and stored at $-20^{\circ} \mathrm{C}$. Iron saturation level was confirmed spectrophotometrically (Bates and Schlabach, 1973; Brisson et al., 2007). Lactoferrin with different iron saturation levels (1.0, 9.0, 38,58 , and $96 \%$ ) were prepared and named as follows: apo-LF, LF-9, LF-38, LF-58, and holo-LF.

\section{Osteoblast-Like Cell Culture}

Osteoblasts were isolated from 24-h fetal rat calvariae, as previously described (Cornish et al., 1999). Briefly, calvariae were excised and the frontal and parietal bones, free of suture and periosteal tissue, were collected. The calvariae were sequentially digested using collagenase, and the cells were collected, pooled, and washed. Cells were grown in T75 flasks in Dulbecco's modified Eagle's medium (HyClone, Beijing, China) with $10 \%$ fetal bovine serum (FBS; PAA, Linz, Austria) for $2 \mathrm{~d}$ and then changed to $\alpha$-modified Eagle's medium (HyClone) plus 10\% FBS and grown to 90\% confluency (Cornish et al., 2004). After trypsinization with trypsin $(0.25 \%)$, the cells were rinsed in $\alpha$-modified Eagle's medium with $10 \%$ FBS and resuspended in fresh medium. The cells were incubated under $5 \% \mathrm{CO}_{2}$ at $37^{\circ} \mathrm{C}$.

\section{Cell Proliferation Assay}

The colorimetric 3-(4,5-dimethylthiazol-2-yl)-2,5diphenyltetrazolium bromide (MTT; Sigma, St. Louis, MO) assay was used to assess the effect of LF on proliferation of primary osteoblasts. Therefore, cells were seeded into 96-well tissue culture plates (Corning Inc., Corning, NY) at a density of $2.5 \times 10^{4}$ cells/ $\mathrm{cm}^{2}$. Twenty-four hours after subculturing, cells were changed to serum-free medium for a further $12 \mathrm{~h}$ before the addition of LF with different iron saturation levels. Fresh media and LF samples at different concentrations $(10,100,1,000 \mu \mathrm{g} / \mathrm{mL})$ were then added for a further $44 \mathrm{~h}$. After the treatment period, MTT $(5 \mathrm{mg} / \mathrm{mL}$ in PBS) was added to each cell. After a 4-h incubation, the supernatant was removed and dimethylsulfoxide (Sigma) was added to solubilize MTT for $4 \mathrm{~h}$. After extraction with dimethylsulfoxide, the optical density was measured at $570 \mathrm{~nm}$ (plate reader, Bio-Rad Laboratories, Hercules, CA). 


\section{In Vivo Study}

Four-week-old ICR Swiss male mice were randomly divided into 4 groups (6 mice per group): blank control (PBS), negative control (BSA), apo-LF, and holo-LF. Four groups of mice were injected subcutaneously with PBS, BSA, apo-LF, or holo-LF, respectively, over the calvarial surface, twice a day for 5 consecutive days at a dosage of $4 \mathrm{mg} / \mathrm{kg}$ per day. To exclude the nutritional effect of LF as a protein, BSA was chosen as a control protein. To measure mineral apposition rate (MAR), double tetracycline- $\mathrm{HCl}$ labeling was performed at $\mathrm{d} 7$ and 14 by intraperitoneal injection $(30 \mathrm{mg} / \mathrm{kg})$. The mice were anesthetized by using isoflurane and then killed by cervical dislocation $7 \mathrm{~d}$ after the second labeling. The labeling was examined in calvarial samples embedded in methyl methacrylate. The dissected calvarial samples were fixed in $75 \%$ ethanol and embedded in methyl methacrylate. Unstained transverse sections (3 mm thick) were examined by using a fluorescence microscope. The MAR was measured and analyzed using Image-Pro Plus software (version 6.0; Media Cybernetics Inc., Rockville, MD; Cornish et al., 2004). All animal procedures were approved by the animal ethics committee of our institution.

\section{Circular Dichroism Measurements}

Circular dichroism (CD) measurements in the farUV region were carried out by using protein solutions in Milli-Q water (Millipore) with appropriate blanks. These measurements were done with a J-810 CD spectropolarimeter (Jasco, Tokyo, Japan). All samples were incubated against Milli-Q water at $4^{\circ} \mathrm{C}$ for $12 \mathrm{~h}$; the protein concentration used was $0.2 \mathrm{mg} / \mathrm{mL}$ and the measurements were made at $25^{\circ} \mathrm{C}$. A cell with a path length of $0.1 \mathrm{~cm}$ was used for the scans between 200 and $260 \mathrm{~nm}$. The estimation of the contents of $\alpha$-helix, $\beta$-structure, and unordered structure was performed according to Yang et al. (1986).

\section{Intrinsic Tryptophan Fluorescence}

Intrinsic fluorescence emission spectra were recorded using a Cary Eclipse Spectrofluorimeter (Varian, Middelburg, the Netherlands) at $25^{\circ} \mathrm{C}$. The protein concentration used was $0.08 \mathrm{mg} / \mathrm{mL}$, and the samples were equilibrated at $25^{\circ} \mathrm{C}$ for $30 \mathrm{~min}$ before fluorescence measurements. Excitation and emission slit widths were kept at $5 \mathrm{~nm}$. Emission spectra were recorded in the range 300 to $400 \mathrm{~nm}$ after excitation with a wavelength of $290 \mathrm{~nm}$. Appropriate blanks were used for the baseline correction of fluorescence intensity (Sreedhara et al., 2010), and measurements were done in triplicate.

\section{Statistics}

The results were expressed as means \pm standard deviations. Data analysis was carried out using SPSS software (version 17.0 SPSS Inc., Chicago, IL). Differences among groups were compared using ANOVA followed by Duncan's test. Statistical significance was defined at a level of $P<0.05$.

\section{RESULTS AND DISCUSSION}

\section{Effect of Iron Saturation Level on the Stimulating Primary Osteoblast Activity of LF}

The MTT assay was used to assess the stimulating proliferation activity of LF with different iron saturation levels at different concentrations. Figure 1 showed that all of the LF samples could stimulate osteoblast proliferation at concentrations of 10,100 , and $1,000 \mu \mathrm{g} /$ $\mathrm{mL}$ compared with control. At $10 \mu \mathrm{g} / \mathrm{mL}$, both apo-LF and holo-LF had stimulatory effects on osteoblast proliferation (Figure 1A), suggesting that iron saturation level had no effect on osteogenic activity of LF at 10 $\mu \mathrm{g} / \mathrm{mL}$. However, with an increase in iron saturation level from 1.0 to $96 \%$, the ratio of osteoblast proliferation decreased from 2.06 to 1.50 at $100 \mu \mathrm{g} / \mathrm{mL}$ (Figure 1B) and decreased from 2.19 to 1.76 at $1,000 \mu \mathrm{g} / \mathrm{mL}$ (Figure 1C). The stimulatory activity on osteoblasts of apo-LF was significantly $(P<0.05)$ higher than that of holo-LF at 100 and $1,000 \mu \mathrm{g} / \mathrm{mL}$, indicating that stimulating proliferation activity was related to the iron saturation level of LF.

\section{LF with Different Iron Saturation Levels Increased Bone Growth In Vivo}

The distance between 2 tetracycline- $\mathrm{HCl}$ labels represented new bone formation over $7 \mathrm{~d}$. The bone growth on calvariae was evaluated directly by the distance between the new bone surface and the old bone. The photomicrographs of calvariae showed that BSA had no effect on bone formation compared with PBS control (data not shown). The distances between 2 tetracycline- $\mathrm{HCl}$ labels of both apo-LF and holoLF were wider than those of controls (Figure 2B and $\mathrm{C})$. The new bone area over the calvarial surface was quantified by MAR. Local injection results showed that both apo-LF and holo-LF significantly increased MAR in vivo compared with control $(P<0.05$; Figure $2 \mathrm{D})$. 
Although the difference was not significant $(P>0.05)$, the effect on new bone growth of apo-LF tended to be stronger than that of holo-LF.

\section{Changes in the Secondary Structure of LF with Different Iron Saturation Levels}

The CD spectra of a protein in the far-UV region (200 to $250 \mathrm{~nm}$ ) were used to monitor conformational changes in the polypeptide backbone. The effect of iron saturation level on the CD secondary structure of LF is shown in Figure 3. The peaks observed at wavelengths 208, 216, and $222 \mathrm{~nm}$ of the 5 samples were different. These 3 peaks of apo-LF were smaller compared with those of holo-LF. We calculated the contents of $\alpha$-helices, $\beta$-structures, turns, and unordered structures of these samples (Table 1 ) and showed that the content of $\alpha$-helices increased with increasing iron saturation level, whereas the content of $\beta$-structures decreased.

\section{Effect of Iron Saturation Level on Intrinsic Tryptophan Fluorescence of LF}

Intrinsic tryptophan fluorescence is used to reflect the unfolding of protein (Gorinstein et al., 2000) and tertiary structural alterations in protein (Liang and Chakrabarti, 1982). The changes in fluorescence intensity of LF with different iron saturation levels are shown in Figure 4. For apo-LF and holo-LF, the emission maximum values were found to be 336 and $343 \mathrm{~nm}$, respectively, and both values increased with increasing iron saturation. At the same time, relative fluorescence intensity decreased from 47 to 17 arbitrary units. Thus, with increasing iron saturation, the fluorescence intensity decreased and shifted red, from 336 to $343 \mathrm{~nm}$.

This study provides direct evidence that osteogenic activity decreased with the increase of iron saturation level in vitro and in vivo and the conformation of LF changed. Because endotoxin is a common contaminant in LF and has been known to influence cell culture work, we used the MTT assay to test whether endotoxin content in LF samples affected osteoblast proliferation. Results showed that the level of endotoxin found $(0.22$ $\times 10^{-3} \mathrm{EU} / \mathrm{mg}$ of LF) had no effect on osteoblast proliferation (data not shown). Jiang et al. (2002) showed that lipopolysaccharide had no effect on the formation of osteoclastic cells at the same level. Therefore, the content of endotoxin in the LF samples did not affect our conclusions.

We found that iron saturation level had no effect on the stimulating proliferation activity of LF at $10 \mu \mathrm{g} /$ $\mathrm{mL}$. This result was in agreement with a published study (Cornish et al., 2006), which indicated that apoLF and holo-LF exerted similar stimulatory effects on
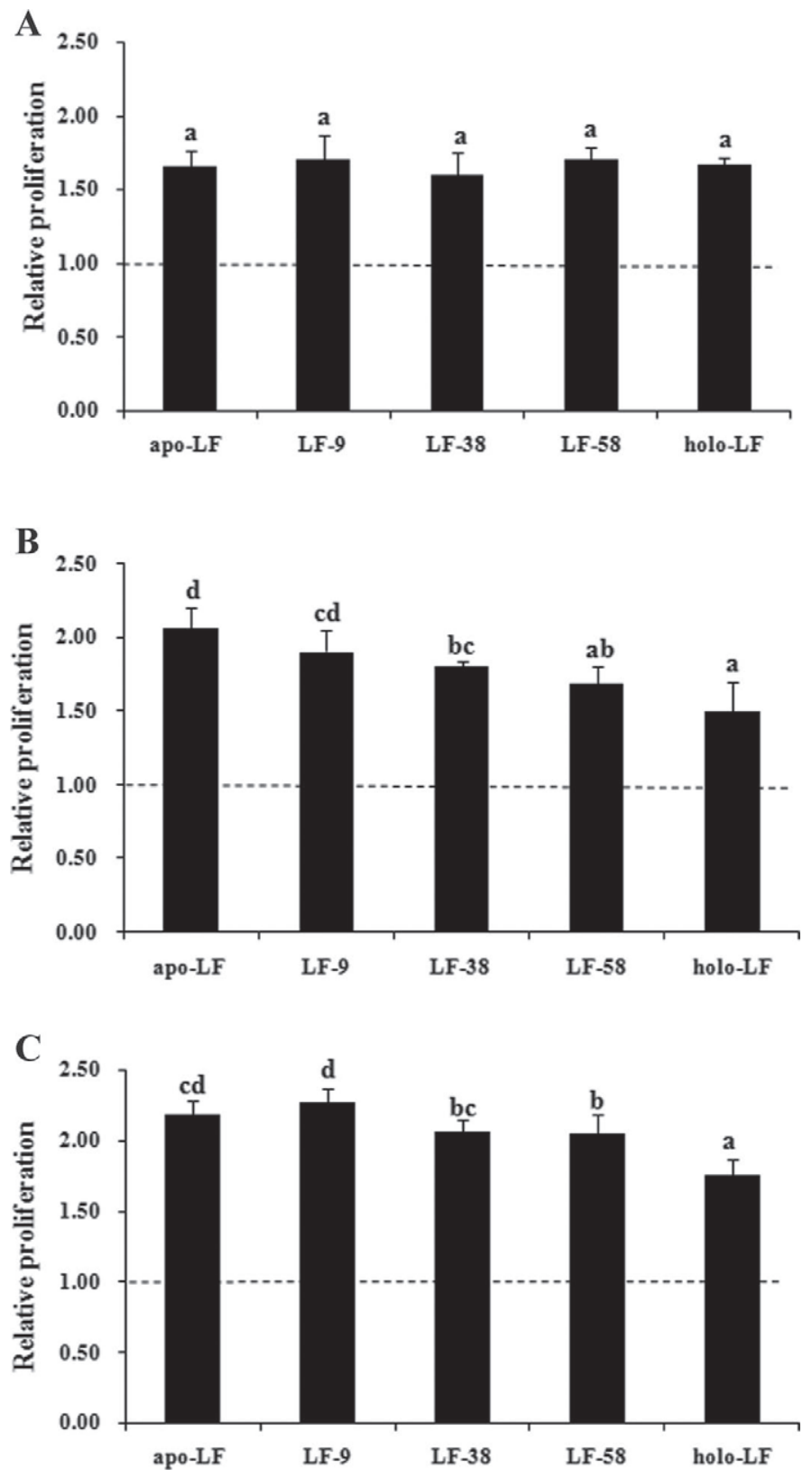

Figure 1. Effect of bovine lactoferrin (LF) with different iron saturation levels on osteoblast proliferation at concentrations of $10 \mu \mathrm{g} /$ $\mathrm{mL}(\mathrm{A}), 100 \mu \mathrm{g} / \mathrm{mL}$ (B), 1,000 $\mu \mathrm{g} / \mathrm{mL}$ (C), assessed using the MTT [3-(4,5-dimethylthiazol-2-yl)-2,5-diphenyltetrazolium bromide] assay. Apo-LF, LF-9, LF-38, LF-58, and holo-LF represent LF with different iron saturation levels $(1.0,9.0,38,58$, and $96 \%)$. Data are presented as treatment:control ratios; the dashed line in each panel represents proliferation in the control. Data are means $\pm \mathrm{SD}(\mathrm{n}=6)$; means without a common letter differ, $P<0.05$.

osteoblast proliferation at 1 and $10 \mu \mathrm{g} / \mathrm{mL}$. However, Cornish et al. (2006) did not evaluate higher concentrations. We found that stimulating proliferation activity decreased with increasing iron saturation level at concentrations of 100 and $1,000 \mu \mathrm{g} / \mathrm{mL}$ and presumed 
A

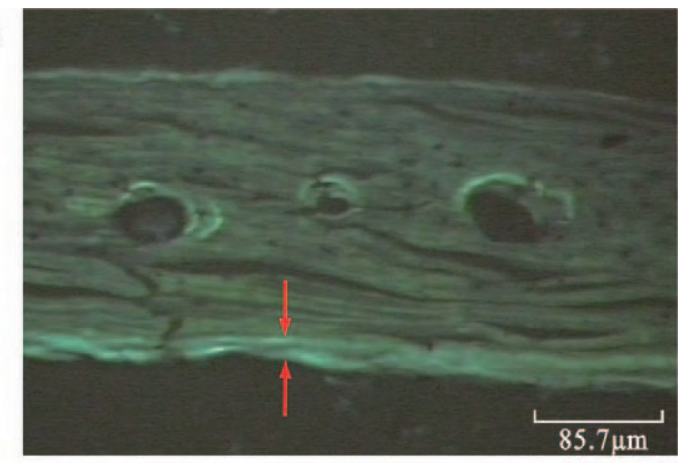

B

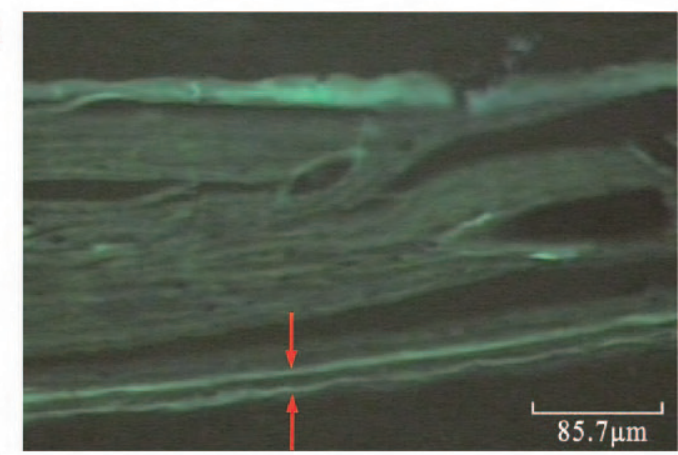

C

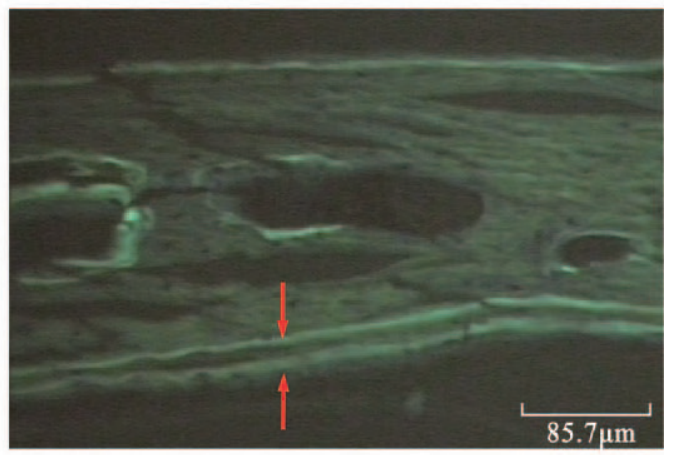

D

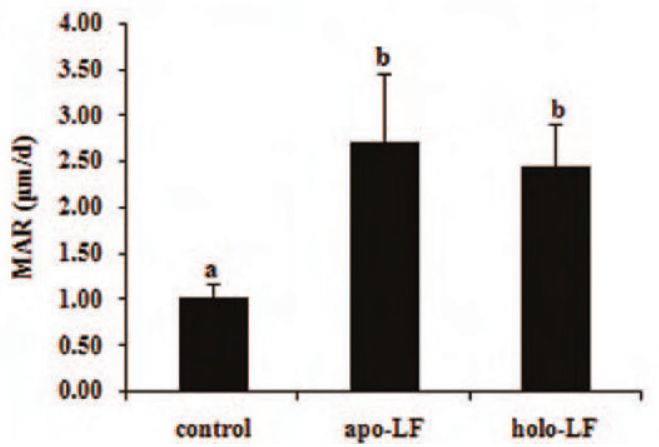

Figure 2. Photomicrographs of calvariae from animals treated with PBS (control; A), apo-lactoferrin (B), and holo-lactoferrin (C) for $5 \mathrm{~d}$. Animals were killed $16 \mathrm{~d}$ later. Tetracycline- $\mathrm{HCl}$ was used as fluorochrome label and given twice, $7 \mathrm{~d}$ apart. The increased new bone growth in the 7-d period (i.e., distance between the 2 tetracycline- $\mathrm{HCl}$ labels, arrows) can be appreciated in the calvariae from the animals treated with apo-LF and holo-LF. Mineral apposition rate (MAR) was quantified $(\mathrm{D})$. Data are means $\pm \mathrm{SD}(\mathrm{n}=6)$; means without a common letter differ, $P<0.05$. Color version available in the online PDF. that the effect of iron saturation level on stimulating proliferation activity of LF may be dose related.

Bone formation in vivo results from a stimulatory effect on osteoblasts and an inhibitory effect on osteoclasts. The stimulating osteoblast proliferation activity does not represent the whole condition of bone growth in vivo. Therefore, bone formation activity was used to confirm the effect of iron saturation level on osteogenic activity of LF in vivo. Our results showed that both apoLF and holo-LF significantly increased bone growth. A previous report of the effect of LF on bone formation (Cornish et al., 2004) showed that LF increased new bone formation through injection over calvariae of adult mice. We confirmed that LF could stimulate bone growth in vivo. Moreover, results of MAR indicated that the new bone formation effect of apo-LF tended to be stronger than that of holo-LF, although the results were not significant. The in vitro results and in vivo results were not in a strict corresponding relationship, and this difference may be caused by the dose or the response time of LF samples in vitro and vivo.

Many studies have reported that the iron saturation level of LF affects its activities except for osteogenic activity. Removal of iron from LF decreases its overall stability and increases accessibility of pepsin to potential cleavage sites of LF (Chan and Li-Chan, 2007). In addition, studies show that the effect of apo-LF on stimulating proliferation of crypt cells is significantly stronger than that of holo-LF (Jiang and Lönnerdal, 2012). The results of the current study pose the ques-

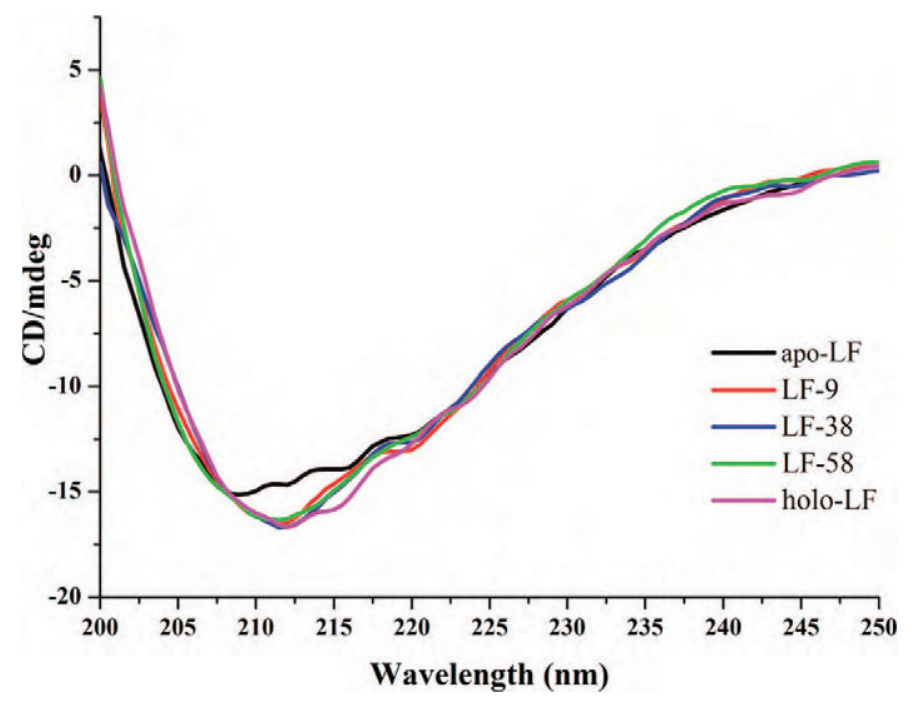

Figure 3. Far-UV circular dichroism (CD) spectra of bovine lactoferrin (LF) with different iron saturation levels. Spectra were obtained at $25^{\circ} \mathrm{C}$ in Milli-Q water (Millipore, Billerica, MA). Apo-LF, LF-9, LF38, LF-58, and holo-LF represented LF with different iron saturation levels $(1.0,9.0,38,58$, and $96 \%)$. The values are given as a mean of 3 parallel scans. Color version available in the online PDF. 
Table 1. Secondary structural contents of lactoferrin (LF) with different iron saturation levels as measured by circular dichroism

\begin{tabular}{lcccc}
\hline $\begin{array}{l}\text { Lactoferrin }_{\text {sample }}^{1} \\
\text { apo-LF }\end{array}$ & $\alpha$-Helix (\%) & $\beta$-Structure (\%) & Turn (\%) & $\begin{array}{c}\text { Unordered } \\
\text { structure (\%) }\end{array}$ \\
\hline LF-9 & 18.0 & 57.1 & 0.2 & 24.6 \\
LF-38 & 19.8 & 56.0 & 0.0 & 24.2 \\
LF-58 & 20.4 & 52.9 & 2.9 & 23.8 \\
holo-LF & 22.5 & 50.6 & 6.2 & 20.8 \\
\hline
\end{tabular}

${ }^{1}$ Apo-LF, LF-9, LF-38, LF-58, and holo-LF represent LF with different iron saturation levels: 1.0, 9.0, 38, 58, and $96 \%$, respectively.

tion why iron release affects the osteogenic activity of LF.

To address this question, we evaluated the conformational changes of LF with iron binding and release. The CD spectra of a protein in the far-UV region (200 to $250 \mathrm{~nm}$ ) were used to monitor conformational changes in the secondary structure. We observed that the content of $\alpha$-helices increased and that of $\beta$-structures decreased with increasing iron saturation level. In accordance with our results, Sreedhara et al. (2010) found that the content of $\alpha$-helix decreased with iron content loss from holo-LF triggered by $\mathrm{pH}$. Furthermore, intrinsic fluorescence spectra provide a sensitive means of characterizing protein conformations. Our results showed that the fluorescence intensity decreased and shifted red from 336 to $343 \mathrm{~nm}$ with the increase in iron saturation level. The red shift indicated that trypto-

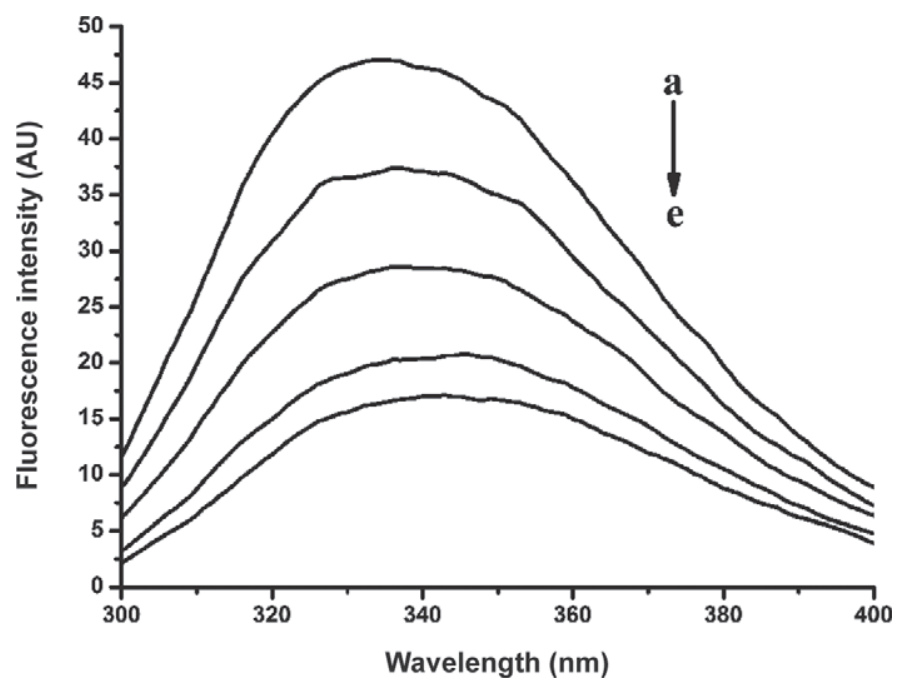

Figure 4. Fluorescence spectra of lactoferrin (LF) with different iron saturation levels (the curves from top to bottom are apo-LF, LF9, LF-38, LF-58, and holo-LF; curves a to e). Apo-LF, LF-9, LF-38, LF-58, and holo-LF represent LF with different iron saturation levels $(1.0,9.0,38,58$, and $96 \%)$. The curves showed fluorescence intensity (arbitrary units, AU) of LF samples at different wavelength. The excitation wavelength was $290 \mathrm{~nm}$, and the emission was scanned from 300 to $400 \mathrm{~nm}$. Samples were incubated for $30 \mathrm{~min}$ at $25^{\circ} \mathrm{C}$. phan was exposed to a more hydrophilic environment. Biophysical studies have demonstrated that apo-LF is less stable and less compact than holo-LF (Grossmann et al., 1992), and x-ray analyses show that, in the structure of apo-LF, the N-lobe is wide open following a $54^{\circ}$ rotation about a hinge behind the iron site, mediated by the pivoting of 2 helices and flexing of 2 interdomain polypeptide strands (Anderson et al., 1990). These results suggest that the conformation of LF tended to be more open with a decrease of iron saturation level.

Grey et al. (2004) found that the mitogenic effect of LF in osteoblast acts through the low-density lipoprotein receptor-related protein 1 , which functions as a signaling receptor in osteoblasts. Our studies showed that tryptophan of LF was exposed to a more hydrophilic environment with the increase of iron saturation level and that the conformation of apo-LF was more open and less compact than that of holo-LF. This conformation difference between LF with different iron saturation levels may result in different parts of LF being exposed to low-density lipoprotein receptor-related protein 1 , resulting in a difference in osteogenic activity between LF with different iron saturation levels.

\section{CONCLUSIONS}

In our study, we evaluated the effect of LF with different iron saturation levels on osteogenic activity in vitro and in vivo. The stimulating osteoblast proliferation activity of LF in vitro decreased with an increase in iron saturation level, and new bone formation ability in vivo followed the same trend. We infer that this may be related to the conformational changes of LF that occur with iron binding and removal, but this finding needs to be explored further.

\section{ACKNOWLEDGMENTS}

This study was supported by National Natural Science Foundation of China (31000771), National Science and Technology Support Program (2012BAD28B07), and Chinese Universities Scientific Fund (2011JS113). 


\section{REFERENCES}

Anderson, B. F., H. M. Baker, G. E. Norris, S. V. Rumball, and E. N. Baker. 1990. Apolactoferrin structure demonstrates ligand-induced conformational change in transferrins. Nature 344:784-787.

Baker, H. M., and E. N. Baker. 2004. Lactoferrin and iron: Structural and dynamic aspects of binding and release. Biometals 17:209216.

Bates, G. W., and M. R. Schlabach. 1973. The reaction of ferric salts with transferrin. J. Biol. Chem. 248:3228-3232.

Blais, A., A. Malet, T. Mikogami, C. Martin-Rouas, and D. Tomé. 2009. Oral bovine lactoferrin improves bone status of ovariectomized mice. Am. J. Physiol. Endocrinol. Metab. 296:E1281E1288.

Brisson, G., M. Britten, and Y. Pouliot. 2007. Heat-induced aggregation of bovine lactoferrin at neutral $\mathrm{pH}$ : Effect of iron saturation. Int. Dairy J. 17:617-624.

Bullen, J. J., H. J. Rogers, and L. Leigh. 1972. Iron-binding proteins in milk and resistance to Escherichia coli infection in infants. BMJ 1:69-75.

Chan, J. C. K., and E. C. Y. Li-Chan. 2007. Production of lactoferricin and other cationic peptides from food grade bovine lactoferrin with various iron saturation levels. J. Agric. Food Chem. 55:493-501.

Cornish, J., K. E. Callon, C. Q. X. Lin, C. L. Xiao, T. B. Mulvey, G. J. S. Cooper, and I. R. Reid. 1999. Trifluoroacetate, a contaminant in purified proteins, inhibits proliferation of osteoblasts and chondrocytes. Am. J. Physiol. Endocrinol. Metab. 277:E779-E783.

Cornish, J., K. E. Callon, D. Naot, K. P. Palmano, T. Banovic, U. Bava, M. Watson, J. M. Lin, P. C. Tong, Q. Chen, V. A. Chan, H. E. Reid, N. Fazzalari, H. M. Baker, E. N. Baker, N. W. Haggarty, A. B. Grey, and I. R. Reid. 2004. Lactoferrin is a potent regulator of bone cell activity and increases bone formation in vivo. Endocrinology 145:4366-4374.

Cornish, J., K. Palmano, K. E. Callon, M. Watson, J. M. Lin, P. Valenti, D. Naot, A. B. Grey, and I. R. Reid. 2006. Lactoferrin and bone; structure-activity relationships. Biochem. Cell Biol. 84:297-302.

Gorinstein, S., I. Goshev, S. Moncheva, M. Zemser, M. Weisz, A. Caspi, I. Libman, H. T. Lerner, S. Trakhtenberg, and O. MartínBelloso. 2000. Intrinsic tryptophan fluorescence of human serum proteins and related conformational changes. J. Protein Chem. 19:637-642.

Grey, A., T. Banovic, Q. Zhu, M. Watson, K. Callon, K. Palmano, J. Ross, D. Naot, I. R. Reid, and J. Cornish. 2004. The low-density lipoprotein receptor-related protein 1 is a mitogenic receptor for lactoferrin in osteoblastic cells. Mol. Endocrinol. 18:2268-2278.
Grossmann, J. G., M. Neu, E. Pantos, F. J. Schwab, R. W. Evans, E. Townes-Andrews, P. F. Lindley, H. Appel, W. G. Thies, and S. S. Hasnain. 1992. X-ray solution scattering reveals conformational changes upon iron uptake in lactoferrin, serum and ovo-transferrins. J. Mol. Biol. 225:811-819.

Guo, H. Y., L. Jiang, S. A. Ibrahim, L. Zhang, H. Zhang, M. Zhang, and F. Z. Ren. 2009. Orally administered lactoferrin preserves bone mass and microarchitecture in ovariectomized rats. J. Nutr. 139:958-964.

Huang, N., D. Bethell, C. Card, J. Cornish, T. Marchbank, D. Wyatt, K. Mabery, and R. Playford. 2008. Bioactive recombinant human lactoferrin, derived from rice, stimulates mammalian cell growth. In Vitro Cell. Dev. Biol. Anim. 44:464-471.

Jiang, R., and B. Lönnerdal. 2012. Apo- and holo-lactoferrin stimulate proliferation of mouse crypt cells but through different cellular signaling pathways. Int. J. Biochem. Cell Biol. 44:91-100.

Jiang, Y., C. K. Mehta, T.-Y. Hsu, and F. F. H. Alsulaimani. 2002. Bacteria induce osteoclastogenesis via an osteoblast-independent pathway. Infect. Immun. 70:3143-3148.

Liang, J. N., and B. Chakrabarti. 1982. Spectroscopic investigations of bovine lens crystallins. 1. Circular dichroism and intrinsic fluorescence. Biochemistry 21:1847-1852.

Lönnerdal, B., and S. Iyer. 1995. Lactoferrin: Molecular structure and biological function. Annu. Rev. Nutr. 15:93-110.

Lönnerdal, B., R. Jiang, and X. Du. 2011. Bovine lactoferrin can be taken up by the human intestinal lactoferrin receptor and exert bioactivities. J. Pediatr. Gastroenterol. Nutr. 53:606-614.

Metz-Boutigue, M. H., J. Jolles, and J. Mazurier. 1984. Human lactotransferrin: Amino acid sequence and structural comparisons with other transferrins. Eur. J. Biochem. 145:659-676.

Nagasawa, T., I. Kiyosawa, and K. Kuwahara. 1972. Amounts of lactoferrin in human colostrum and milk. J. Dairy Sci. 55:1651-1659.

Sánchez, L., P. Aranda, M. D. Perez, and M. Calvo. 1988. Concentration of lactoferrin and transferrin throughout lactation in cow's colostrum and milk. Biol. Chem. Hoppe Seyler 369:1005-1008.

Sawyer, L., and C. Holt. 1993. The secondary structure of milk proteins and their biological function. J. Dairy Sci. 76:3062-3078.

Sreedhara, A., R. Flengsrud, T. Langsrud, P. Kaul, V. Prakash, and G. E. Vegarud. 2010. Structural characteristic, $\mathrm{pH}$ and thermal stabilities of apo and holo forms of caprine and bovine lactoferrins. Biometals 23:1159-1170.

Yang, J. T., C. S. C. Wu, and H. M. Martinez. 1986. Calculation of protein conformation from circular dichroism. Methods Enzymol. 130:208-269. 